\title{
Thermal Fluctuations of Bismuth Based 1G Tape
}

\author{
M. Chrobak, W.M. Woch*, R. Zalecki and A. KoŁodziejczyk \\ AGH University of Science and Technology, Faculty of Physics and Applied Computer Science, \\ Solid State Physics Department, al. A. Mickiewicza 30, 30-059 Cracow, Poland
}

\begin{abstract}
The thermal fluctuations of bismuth based commercial $1 \mathrm{G}$ tape were studied near the critical temperature $T_{\mathrm{c}}=110.2 \mathrm{~K}$. The detailed analysis of the temperature dependence of resistivity measurements was made in the temperature region from the zero resistance critical temperature up to $300 \mathrm{~K}$. The thermal fluctuations of conductivity were analysed using the Aslamazov-Larkin microscopic approach and the critical exponents were calculated close to the transition temperature.
\end{abstract}

DOI: 10.12693/APhysPolA.127.306

PACS: 74.72.-h, 74.78.-w, 74.40.-n, 74.62.-c

\section{Introduction}

The high temperature superconductors (HTS) possess special features in comparison to the classical ones. HTS are extreme second type with the large penetration depth $\lambda$ of the order of $10^{3}-10^{4} \AA[1]$ and very small coherence length $\xi$ of the order of $10 \AA$ [2]. That makes the Ginzburg-Landau parameter $\kappa=\lambda / \xi$ of the order of $10^{2}$ or more. Other important superconducting parameters are also extremely anisotropic (e.g. the ratio of $c$ - to $a b-$ direction of penetration depths $\gamma=\lambda_{c} / \lambda_{a b}$ is of the order of $10-10^{2}$ for YBCO and of the order of $10^{4}$ for BSCCO) like the critical currents, the irreversibility fields and others, because of the anisotropic layered structure of weakly coupled $\mathrm{CuO}_{2}$ superconducting planes. As a consequence the huge thermal fluctuations around the critical temperature of the superconducting transitions in HTS are observed. Within this critical region the competition between the critical fluctuations at lower temperatures and the Gaussian (stochastic) fluctuations at the temperatures above the critical temperatures exists and depends on the applied magnetic field as well as the applied pressure [3-5]. Because of the huge anisotropy the superconductors exhibit generally the three-dimensional (3D) to the two-dimensional (2D) transition in the critical region for polycrystalline, single crystal as well as for thin film samples [6-9]. There are also observed the crossover from $2 \mathrm{D}$ to $1 \mathrm{D}$ in $\mathrm{Hg}$-Tl 1223 polycrystalline superconductor [10]. In this paper the thermal fluctuations of the $1 \mathrm{G}$ commercial BSCCO tape in the superconducting transition were studied. The critical exponents were obtained as well.

\section{Experimental}

The commercial first generation bismuth based superconducting tape was manufactured by the American Superconductor Company (AMSC). The commercially

\footnotetext{
* corresponding author; e-mail: wmwoch@agh.edu.pl
}

identifying of this tape is as follows: $1 \mathrm{G}$ High Strength Plus 135A Ic min "5715 135" BSCCO.

The temperature dependences of the resistance were measured using the standard four-point ac method to take advantage from Stanford SR 830 lock-in nanovoltometer. The temperature from $77 \mathrm{~K}$ to $300 \mathrm{~K}$ was monitored by a Lake Shore temperature controller with applied a chromel-gold- $0.07 \%$ Fe thermocouple with an accuracy of $\pm 0.05 \mathrm{~K}$. The electrical contacts were soldered with tin. The distance between the voltage contacts was $2 \mathrm{~cm}$. The current applied during the measurements was $8 \mathrm{~mA}$. Each data point is the average from the 25 time repetition.

\section{Results and discussion}

The thermal fluctuations of $1 \mathrm{G}$ BSCCO tape were studied on the basis of the temperature dependences of the resistance that is shown in Fig. 1. The critical parameters of this sample are as follows: the zero resistance critical temperature $T_{\mathrm{c} 0}=109.8 \mathrm{~K}$ that was obtained using the $1 \mu \mathrm{V} / \mathrm{cm}$ electrical field criterion, $T_{\mathrm{c} 50 \%}=110.2 \mathrm{~K}$ and $T_{\text {c_onset }}=111.6 \mathrm{~K}$. We also obtained the transition width which is $\Delta T_{90 \%-10 \%}=0.7 \mathrm{~K} . T_{\mathrm{c}}=110.6 \mathrm{~K}$, respectively. This indicates that the transition to the superconducting state is three-step process. The position $T_{\mathrm{A}}$ corresponds to the weak intergranular links and the zero resistance critical temperature. The position $T_{\mathrm{B}}$ corresponds to the grains and the $T_{\mathrm{c} 50 \%}$ critical temperature and finally the position $T_{\mathrm{c}}$ is related to the fluctuations near onset superconducting transition.

The analysis of thermal fluctuations has been made on the basis of the following formula [11]:

$$
\Delta \sigma=K \varepsilon^{-\lambda},
$$

where $\sigma$ is the temperature dependence conductivity, $\varepsilon=\left(T-T_{\mathrm{c}}\right) / T_{\mathrm{c}}, \lambda$ is a critical exponent and $K$ is a constant. The temperature dependence of excess conductivity is defined within the Ginzburg-Landau mean field approximation as

$$
\Delta \sigma(T)=\frac{1}{R(T)}-\frac{1}{R_{\mathrm{R}}(T)},
$$




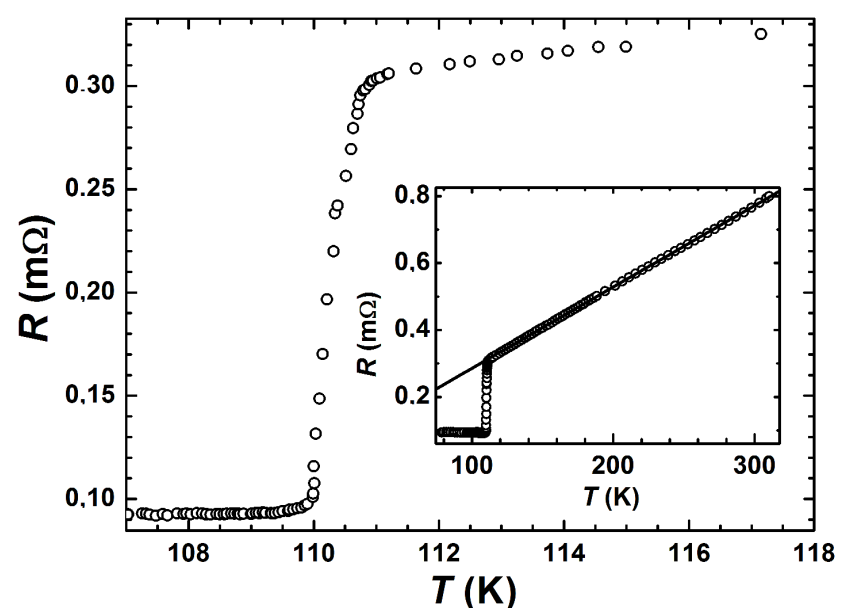

Fig. 1. Resistance versus temperature of BSCCO tape using the applied current of $8 \mathrm{~mA}$. Inset: the resistance data from room temperature down to the onset temperature that was fitted by the linear function (see text).

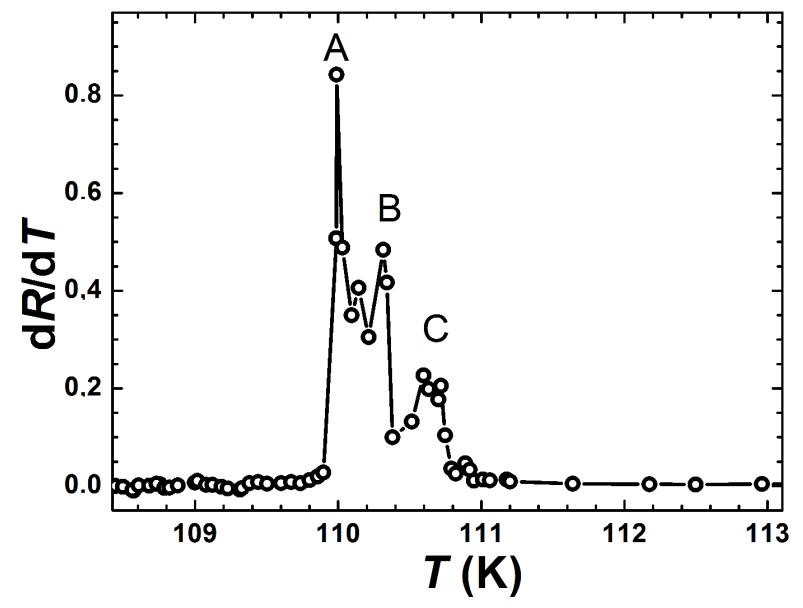

Fig. 2. Derivative of the resistance as a function of temperature. There are characteristic temperatures related to the three peaks: A, B and C described in the text.

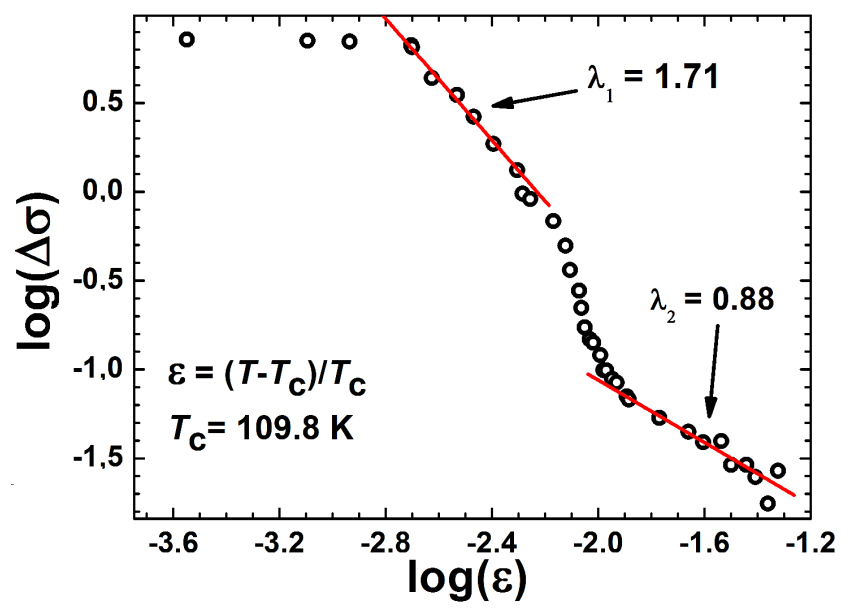

Fig. 3. $\log (\Delta \sigma)$ vs. $\log (\varepsilon)$ dependence of BSCCO tape with lines fitted to linear regions. The zero resistance critical temperature $T_{\mathrm{c} 0}=109,8 \mathrm{~K}$ was used to calculate $\varepsilon$. where $R(T)$ is the measured resistivity and $R_{R}(T)$ is the resistivity obtained by the linear extrapolation of the resistivity data from room temperature down to the onset temperature (see inset in Fig. 1). To acquire the $\Delta \sigma$ it is necessary to determine $R_{\mathrm{R}}(T)$ which is made by extrapolating normal state behaviour as follows:

$$
R_{\mathrm{R}}(T)=R_{0}+\left(\frac{\mathrm{d} R}{\mathrm{~d} T}\right) T
$$

where $R_{0}$ and $\mathrm{d} R / \mathrm{d} T$ are constants. $R_{0}$ is the intercept of line fitted to measured data in high temperatures. The way to determine the critical exponents was described in detail in the papers $[3,5,12]$.

To obtain values of critical exponents $\lambda$ Eq. (1) was transformed into the following formula:

$$
\log \Delta \sigma=-\lambda \log \varepsilon+C,
$$

where the searched value of critical exponent $\lambda$ is equal to negative value of the slope of the fitted line to the linear parts of the dependence of Eq. (4). The dependence expressed by Eq. (4) with lines fitted to linear regions is shown in Fig. 3. The zero resistance critical temperature $T_{\mathrm{c} 0}=109.8 \mathrm{~K}$ was used to calculate argument $\varepsilon$.

In our analysis of thermal fluctuations two regimes have been distinguished where power law behaviour was very distinct. First regime was defined from $110 \mathrm{~K}$ to $110.3 \mathrm{~K}$ and second one from $111.2 \mathrm{~K}$ to $114.1 \mathrm{~K}$. The obtained values of critical exponents are $\lambda_{1}=1.71$ and $\lambda_{2}=0.88$, respectively.

The critical exponent $\lambda_{1}$ placed in the critical region corresponds with the critical fluctuations. For the critical region the full scaling theory [13] predicts that the fluctuation conductivity diverges at $T_{\mathrm{c} 0}$ with exponent

$$
\lambda=\nu(2+z-d+\eta),
$$

where $\nu$ is the critical exponent for the coherence length, $z$ is the dynamical exponent, $d$ is the dimensionality of fluctuations spectrum and $\eta$ is exponent for the order parameter of the correlation function. According to the paper [14] we can substitute the following values: $\nu \approx 2 / 3$, $z \approx 3 / 2$ and $\eta \approx 0$ to Eq. (5) in critial fluctuations region. If we put the $\lambda_{1}=1.71$ in this equation we get $d=0.94$. This substitution results in a $1 \mathrm{D}$ fluctuating system.

The second regime is located above the zero critical temperature $T_{\mathrm{c} 0}$ and in accordance with literature $[5,12]$ it is dominated by Gaussian fluctuations. In this case the mean field Ginzburg-Landau theory [11] predicts $\nu \approx 1 / 2, z \approx 2$ and $\eta \approx 0$. Thus Gaussian fluctuations can be described with the following formula:

$$
\lambda=2-\frac{d}{2} .
$$

After using Eq. (6) for the values $\lambda_{2}=0.88$ the dimension of fluctuations was calculated to be $d=2.24$. It means that in this region there is also $2 \mathrm{D}$ fluctuating system. One can say that there is crossover from $2 \mathrm{D}$ to $1 \mathrm{D}$ fluctuating system. The similar crossover was observed in the polycrystalline $(\mathrm{Hg}, \mathrm{Tl}) 1223$ superconductors and described in paper [10]. The authors contended that the appearance of $1 \mathrm{D}$ fluctuating system suggests 
the existence of conducting channels in the superconductors. In the case of bismuth based superconductors the dimensional behaviour depends mainly on their stoichiometry. For Bi 2212 superconductors there is practically two-dimensional system [15], whereas for Bi 2223 superconductors some authors [16] find two-dimensional behaviour while others [17] propose the crossover from $2 \mathrm{D}$ to $3 \mathrm{D}$ fluctuations. Our current study of bulk Bi 2223 superconductor [18] shows that the calculated critical exponent close to the zero critical temperature was found to be $\lambda=1.75$. This exponent is very close to that reported in this paper.

\section{Conclusions}

The temperature dependence of the resistance was carried out for $1 \mathrm{G}$ BSCCO commercial tape. From the measurement we obtained base superconducting parameters which are as follows: $T_{\mathrm{c} 0}=109.8 \mathrm{~K}, T_{\mathrm{c} 50 \%}=110.2 \mathrm{~K}$ and $T_{\text {c_onset }}=111.6 \mathrm{~K}$.

The critical exponents of measured sample have been calculated in the temperatures range from the zero resistance critical temperature to onset temperature. At the temperatures range in the vicinity of the zero critical temperature $T_{\mathrm{c} 0}$ the critical exponent $\lambda_{1}=1.71$ was calculated and $1 \mathrm{D}$ fluctuating system was recognized. In the temperature range from $111.2 \mathrm{~K}$ to $114.1 \mathrm{~K}$ the critical exponent $\lambda_{2}=0.88$ was obtained and $2 \mathrm{D}$ fluctuating system appeared.

\section{Acknowledgments}

This work was supported by the Polish Ministry of Science and Higher Education and its grants for Scientific Research. One of us (M.Ch.) has been partly supported by the EU Human Capital Operation Program, Polish Project No. POKL.04.0101-00-434/08-00.

\section{References}

[1] Z. Ma, R.C. Taber, L.W. Lombardo, A. Kapitulnik, M.R. Beasley, P. Merchant, C.B. Eom, S.Y. Hou, J.M. Phillips, Phys. Rev. Lett. 71, 781 (1993).
[2] See for instance: High Temperature Superconductivity 1, Materials, Ed. A.V. Narlikar, Springer-Verlag, Berlin 2004.

[3] R.M. Costa, P. Pureur, M. Gusmão, S. Senoussi, K. Behnia, Phys. Rev. B 64, 214513 (2001).

[4] L. Mendoça Ferreira, P. Pureur, H.A. Borges, P. Lejay, Phys. Rev. B 69, 212505 (2004).

[5] P. Pureur, R. Menegotto Costa, P. Rodrigues, J. Schaf, J.V. Kunzler, Phys. Rev. B 47, 11420 (1993.

[6] D.H. Kim, K.E. Gray, M.D. Trochet, Phys. Rev. B 45, 10801 (1992).

[7] H.M. Duan, W. Kiehl, C. Dong, A.W. Cordes, M.J. Saeed, D.L. Viar, A.M. Hermann, Phys. Rev. B 43, 12925 (1991).

[8] W.M. Woch, M. Chrobak, A. Kołodziejczyk, Acta Phys. Pol. A 118, 389 (2010).

[9] N.H. Ahmad, N.A. Khan, A.K. Yahya, J. Alloys Comp. 492, 473 (2010).

[10] S.H. Han, J. Axnäs, B.R. Zhao, Ö. Rapp, Phys. C Supercond. 408-410, 679 (2004).

[11] L.G. Aslamazov, A.I. Larkin, Sov. Phys.-Solid State 10, 875 (1968); A.I. Larkin, A.A. Varlamov, in: $S u$ perconductivity, Eds. K.H. Bennemann, J.B. Ketterson, Vol. 1, Springer-Verlag, Berlin 2008, p. 369.

[12] A. Mohanta, D. Behera, Phys. C Supercond. 470, 295 (2010).

[13] P.C. Hohenberg, B.I. Halperin, Rev. Mod. Phys. 49, 435 (1977).

[14] C.J. Lobb, Phys. Rev. B 36, 3930 (1987).

[15] F. Vidal, J.A. Veria, J. Maza, J.J. Ponte, F. GarciaAlvarado, E. Moran, J. Amador, C. Cascales, A. Castro, M.T. Casais, I. Rasines, Physica C 156, 807 (1988).

[16] See for instance: G. Balestrino, A. Nigro, R. Vaglio, M. Marinelli, Phys. Rev. B 39, 12264 (1989).

[17] See for instance: P. Clippe, Ch. Laurent, S.K. Patapis, M. Ausloos, Phys. Rev. B 42, 8611 (1990).

[18] W.M. Woch, M. Chrobak, R. Zalecki, A. Kołodziejczyk, Acta Phys. Pol. A 126, 328 (2014). 